\title{
Détournements naturalistes et horrifiques dans Dingo d'Octave Mirbeau et Cujo de Stephen King
}

\author{
Anna Gural-Migdal ${ }^{1}$ \\ University of Alberta
}

\begin{abstract}
Résumé : Dingo (1913) d'Octave Mirbeau et Cujo (1981) de Stephen King ont en commun d'avoir pour héros un chien dont la transmutation subversive s'effectue en fonction de données naturalistes et horrifiques. Bien que tous deux soient des chiens tueurs, leur violence meurtrière se fonde sur le régime du libre-arbitre pour l'un et du déterminisme pour l'autre. Le but de cette étude est de montrer par le biais de la représentation de ces deux chiens, que l'horrifique chez Mirbeau sert à déconstruire le naturalisme et à en faire vaciller les assises. Si Dingo succombe à sa race et à son milieu, c'est parce qu'il affirme sa volonté de les renier. C'est donc à lui plutôt qu'au narrateur de faire le choix de sa propre fin et d'imposer son histoire contre une autre possible, un récit d'horreur où il serait torturé pour cause de carnage. C'est donc aussi une liberté créatrice qui s'affirme à travers Dingo, tandis que Cujo, lui, n'a d'autre choix que d'être éliminé pour obéir aux lois du genre horrifique, d'autant plus contraignantes chez Stephen King qu'elles sont assujetties à un naturalisme où priment les nécessités du réel.
\end{abstract}

Mots-clés: Déterminisme. Naturalisme. Parodie. Octave Mirbeau. Stephen King.

Nombreux sont les films ayant pour héros un chien fou. Ils s'apparentent à des sousgenres horrifiques spécifiques comme ceux de l'animal tueur ou encore de l'éco horreur. Le film d'éco horreur est particulièrement populaire en Australie, pays très concerné par les questions écologiques et dont est originaire le dingo. Ce chien sauvage en voie d'extinction a d'ailleurs fait l'objet d'un film de Fred Schepisi, Evil Angels (1998) dont l'histoire est tirée d'un événement réel qui avait alors suscité une réaction viscérale au sein de la population australienne. Après la disparition mystérieuse d'un bẻbé que ses parents avaient amené avec eux en camping, la mère fut accusée d'infanticide pour la seule raison qu'elle soutenait avoir $\mathrm{vu}$ un dingo enlever son enfant. L'opinion publique refusant de croire que cet animal emblématique de l'espace australien et de sa conquête masculine puisse s'attaquer à un

\footnotetext{
${ }^{1}$ Anna Gural-Migdal é professora do Departamento de Línguas Modernas e Estudos Culturais na Universidade de Alberta, Canadá, onde leciona Literatura Francesa e Cinema. Especialista de renome internacional em Émile Zola e o Naturalismo, Anna Gural-Migdal realiza pesquisas sobre a literatura do século XIX francês, semiótica, teoria literária e teoria do cinema. Sua pesquisa ressalta o valor dos estudos interdisciplinares, especialmente a relação entre literatura e cinema. Presidente da Associação Internacional Émile Zola e o Naturalismo (AIZEN) e editora da International Review Excavatio, Anna Gural-Migdal publicou mais de uma centena de artigos sobre Zola, Huysmans, Mirbeau, Artaud, Duras, literatura francófona de Quebec e do Magrebe, cultura popular, além de cinema italiano, francês e canadense. Em setembro de 2013, o livro Re-Reading Zola and Worldwide Naturalism foi lançado em homenagem ao seu trabalho à frente da AIZEN, por quase 20 anos. Atualmente está concluindo um livro intitulado Naturalisme et horreur dans la fiction d'Octave Mirbeau, que será publicado na
} França. 
humain, on a préféré diaboliser la femme plutôt que la bête. Le dingo est toutefois devenu une figure de la monstruosité lorsque, preuve à l'appui, on a constaté que cet animal avait bel et bien tué le bébé. Cela a eu pour effet d'entraîner une confusion dans la perception du dingo et de son statut légal. Comme le soulignent James Gorman et Christine Kennealy, "in some parts of Australia dingoes are pests, but in other parts they are protected. Their status can change with shifts in public opinion" (GORMAN; KENNEALY, 06 March 2012, p. D1). De tels faits nous permettent de constater que le dingo n'est pas seulement un être de chair et de sang, mais aussi une construction culturelle, sociale et imaginaire, dotée d'une part de symbolisme voire d'affabulation, ne serait-ce que parce que l'animal se donne à percevoir dans la relation qu'il a avec l'homme et la civilisation.

C'est à partir d'une telle dynamique relationnelle que Jack London a par exemple construit ses deux romans naturalistes, The Call of the Wild (1903) et White Fang (1906) qui inversent deux parcours de vie chez la bête, l'un progressif et l'autre régressif, selon qu'elle accepte ou non la loi des hommes. On ne saurait toutefois considérer l'évolution de Fang comme seul résultat d'un déterminisme du milieu ou d'une éducation éthologique, puisque l'animal finit par transcender l'espèce canine pour devenir le symbole d'un être supérieur qui s'élève au-dessus de la masse des hommes. De même dans The Call of the Wild, le retour de la bête à sa nature brute ne résulte pas uniquement des instincts ou de "l'appel sauvage" car Buck se transforme en héros de légende à la fin du roman. Earle Labor dit à ce sujet: “(...) and Buck is released from the bond of love to fulfill the last phase of his apotheosis as he is transformed into the immortal Ghost Dog of Northland legend; he incarnates the eternal mystery of creation and life" (LABOR, 1983, p. 123). Ainsi, dans les deux cas, la représentation naturaliste de l'animal et de sa lutte pour la survie lui concède paradoxalement une dimension héroïque ou mythique qui revêt un caractère surnaturel. Et si l'auteur glorifie le chien-loup en l'associant aux forces du bien, il n'en demeure pas moins qu'il peut en offrir une perception opposée en le diabolisant comme c'est le cas dans la nouvelle "Bâtard" (1902), où l'animal victime des traitements cruels de son maître devient l'incarnation même du Mal. Habité par la rage et l'esprit de vengeance, il finit par tuer son tortionnaire avant d'être tué à son tour, le naturalisme du texte venant ici déboucher sur l'épouvante.

À partir de telles prémisses, nous nous proposons d'établir une comparaison entre les romans Dingo (1913) d'Octave Mirbeau et Cujo (1982) de Stephen King, qui ont en commun d'avoir pour héros un chien dont la transmutation subversive s'effectue en fonction de données naturalistes et horrifiques. Il nous semble que chez l'auteur américain, le naturalisme 
permet de cautionner la représentation de l'horreur et de sa signification par un contexte réaliste et civilisationnel, tandis que chez Mirbeau les détournements horrifiques visent au contraire à saper la causalité naturaliste du texte pour mettre de l'avant sa part d'invention et de fantaisie. Bien que l'itinéraire narratif des deux chiens se conclue par la mort, celle-ci n'a pas le même fondement car elle repose sur le régime du libre-arbitre pour Dingo et du déterminisme pour Cujo. Nous souhaiterions montrer que ce dernier est contraint de suivre une logique naturelle de déshumanisation en fonction du progrès de sa maladie, alors que Dingo fait montre d'un héroïsme suicidaire en choisissant de faire obstacle à la nature et de s'humaniser pour déjouer toute forme d'explication rationnelle. Cette étude se propose également de souligner la manière dont l'horrifique met à contribution le visuel tant chez King que Mirbeau. Ce dernier esthétise Dingo, il le construit comme image fantastique et nous en donne une vision artistique qui se rattache aussi bien à la peinture qu'au spectacle de l'horreur exquise. ${ }^{2}$

King, de son côté, fait de nombreux emprunts au gothique rural, un sous-genre spécifique à la culture populaire américaine, et de plus certaines scènes de Cujo sont des références directes à des films d'horreur mettant en vedette un animal-monstre.

La fiction de Stephen King se caractérise par une généricité hybride où l'horreur emprunte à la fois à la tradition littéraire du gothique, du réalisme et du naturalisme. Le romancier convoque de surcroît une culture cinématographique qui renvoie, comme l'indique Linda Badley, aux films d'horreur classiques des années 1930 et 1950 (BADLEY, 1996, p. 2). Selon elle, cet aspect filmique de l'horreur chez King, conjointement à son caractère naturaliste, confère toute son originalité à l'œuvre du romancier. Douglas Winter corrobore que le maître de l'horreur contemporaine a été fortement influencé par des écrivains naturalistes tels que Thomas Hardy, Jack London mais surtout Theodore Dreiser, le titre du roman Carrie faisant directement référence à Sister Carrie (WINTER, 1986, p. 23). La poétique de l'horreur chez King réactive donc tout en les modernisant des données à la fois naturalistes et gothiques pour opérer un mélange de réel et de surréel. Jeanne Campbell Reesman note qu'il y a dans l'œuvre de cet écrivain la coexistence ironique d'un surnaturalisme qui résulte de l'horreur et d'un naturalisme qui ressortit à la réalité (REESMAN apud STRENGELL, 2005, p. 183). En teintant son gothique de mythique, de merveilleux et de fantastique, King cherche à transcender les limites des forces de l'hérédité et du milieu, ce qui, le plus souvent, a pour effet paradoxal de les amplifier. De même essaie-

\footnotetext{
${ }^{2}$ Cet oxymoron fait référence à Baudelaire. 
t-il de dépasser les lois de la nature en instaurant une herméneutique du doute qui vise à défier un savoir rationnel par une connaissance révélée, mais qui ironiquement nous ramène à la certitude du Mal. Adaptant la méthode des naturalistes, il tente de comprendre le vivant à partir du mort, le sain en fonction du pathologique, le fonctionnement à même le dysfonctionnement. D'où l'importance du morbide dans sa démarche qui, par le biais de l'horreur, rénove des topoï et des thèmes propres au naturalisme tel que celui du rapport au corps qui détermine la constitution du sujet et sa définition, car se définir vivant, c'est d'abord se définir comme matière vivante et enveloppe corporelle. Dans une telle perspective, le corps est une entité habitée par l'identité puisqu'il y a reconnaissance de soi ou absence à soi à travers lui.

Dans Cujo, le métissage des genres contribue à la représentation horrifique du chien et à l'appréhension de sa monstruosité en lui conférant une corporèité hybride d'où émane une inquiétante ètrangeté: "Pendant une fraction de seconde, il se demanda bêtement s'il s'agissait véritablement d'un chien, ou bien de quelque étrange race de poneys. (...) Vic remarqua ses yeux tristes et comprit que c'était un saint-bernard" (KING, 1981, p. 26). Cette hybridation donne aussi à voir la vie comme forme redoublée ou altérée de la mort. En atteste la morsure de chauve-souris qui transmet le virus de la rage au saint-bernard, le condamnant à la maladie et à la furie. Il s'instaure une textualité du corps où la balafre sur le museau du chien s'écrit sous forme de point d'interrogation comme un scalpel qui opère à vif pour rendre visible le travail souterrain appelé à transformer l'animal. L'explication médicale afférente au naturalisme se double ici d'un procédé visuel qui nous ramène au cinéma d'horreur et fait écho à ce que Jacques Aumont appelle la "dé-visagéification”. Il dit à ce sujet: "À défaut de faire disparaître ou d'altérer le visage en jouant sur les tensions intérieures, on peut l'attaquer de l'extérieur, le gratter, matérialiser dans une procédure graphique ou plastique, rageuse ou appliquée, une blessure, ou parfois sa cicatrice" (AUMONT, 1992, p. 164). La morsure de chauve-souris est la marque du destin qui s'inscrit génètiquement dans le saint-bernard puisqu'elle en modifie l'espèce par la contamination d'une autre espèce, comme si le chien devenait le résultat de ce qu'il avait mangé. À cette perte identitaire qui est aussi perte de soi, point d'interrogation convoquant un vide terminologique, s'ajoute le saccage des traits, cette expression faciale devenue rictus pour anticiper le devenir monstre de l'animal.

L'hybridation de genre et de mode contribue également à souligner une vision proleptique du mort-vivant en conférant à Cujo, dès le début du roman, un caractère fantomatique et fantasmatique. Le récit commence ironiquement comme un conte d'enfant, 
puis se teinte d'horrifique avec l'évocation du serial killer pour ensuite offrir une explication naturaliste ayant trait à la névrose. La réincarnation du tueur en série détraqué Frank Todd, dans Cujo, est véhiculée par le monde de l'enfance, à travers les visions prémonitoires de Tad Trenton. Au lieu de son ours en peluche rassurant, le petit garçon voit en effet apparaître dans son placard une créature effrayante aux yeux semblables à "des trous d'ambre rouge", "une chose qui aurait pu être mi-homme, mi-loup" (KING, 1981, p. 13) dont la tête penchée évoque "l'attitude interrogatrice des prédateurs" (KING, 1981, p. 14). Comme on le sait, l'animal en général ne se définit pas seulement dans la perception qu'on en a, mais ausssi dans le lien de confiance qui s'établit entre lui et l'être humain. Ainsi lorsque Donna, la mère de Tad voit Cujo pour la première fois, elle ne peut s'empêcher d'avoir un mouvement de recul, attrapant instinctivement son fils pour le protéger. Mais Brett Camber, le jeune garçon qui est le maître du chien, la rassure: "Vous pouvez le laisser, madame, dit poliment le garçon. Cujo adore les gosses. Il ne lui fera pas de mal” (KING, 1981, p. 27). Toutefois, le lien entre le monde de l'enfance et le saint-bernard sera brisé par un détail, une plaie saignante, une fêlure qui s'amplifiera dans le cerveau du chien pour le rendre étranger à son jeune maître. À cet égard, Brett vient faire écho à la vision prémonitoire de Tad quand, dans une mise en scène fantastique teintée de merveilleux, il voit son chien surgir comme une apparition, ce qui provoque en lui une réaction de frayeur:

Comme un petit garcon qui se trouva plongé en plein conte de fée, sa première pensée fut: un loup, et il jeta autour de lui des regards affolés. Il ne put rien discerner d'autre que du blanc.

Cujo jaillit du brouillard. (...) Ce chien n'avait que peu de ressemblance avec l'apparition boueuse et hirsute qui se matérialisait lentement dans la brume matinale (KING, 1981, p. 150).

Tel que le constate Pierre Ancet, en ce qui concerne le corps monstrueux, "tout excès contenu dans l'image contient en lui son opposé, c'est-à-dire un repli de l'identité sur ellemême: que le monstre soit visible, en pleine lumière n'y change rien, comme si l'abondance de détails dėgageait une sorte d'invisibilité, d'opacité dans le visible" (ANCET, 2006, p. 20). C'est sur un tel oxymore que se construit le régime visuel de la représentation de Cujo en tant que monstre prédateur. Ainsi la rationalité du monde adulte et l'imagination du monde de l'enfance convergent dans la rage de Cujo. C'est un chien qui souffre d'une vraie maladie, mais c'est aussi une construction imaginaire, la manifestation du monstre archétypal et mythique qui hante les cauchemars des enfants. On peut donc dire qu'il y a un Cujo d'avant et d'après la morsure qui, dans un renversement, fait s'estomper les contours de l'enfance:

Il fut un temps où il aimait le GARÇON, où il serait mort pour lui si on le lui avait demandé. Ce sentiment restait assez fort pour repousser l'idée de tuer 
jusqu'à ce qu'il devint aussi opaque que le brouillard. (...) Ce qui subsistait de l'animal sain, celui d'avant la blessure, se retourna, et avec lui le chien contaminé, dangereux, qui pour la dernière fois se voyait contraint d'obéir (KING, 1981, p. 151).

Cujo et les deux enfants ont en commun d'être tous trois victimes d'un milieu familial dysfonctionnel qui les fera basculer dans le monde adulte et aura pour conséquence de les faire mûrir prématurément, ainsi que d'entraîner la mort du saint-bernard et de Tad. Deux situations initiales réalistes mises en parallèle influent sur le sort de ces personnages. Il y a celle de deux mariages qui sont des échecs. Vic, le père de Tad, apprend que sa femme Donna l'a trompé, ce qui rend incertain l'avenir de leur couple. Charity, la mère de Brett, a épousé une brute qui la bat, et elle rêve d'échapper aux chaînes du mariage. Comme conséquence de ces crises conjugales, il y deux abandons: Brett Camber part avec sa mère pour rendre visite à sa tante dans le Connecticut. De même, Vic Trenton abandonne son enfant en allant à New York afin de sauver sa campagne de publicité pour céréales. La grande ville est évidemment associée au monde de la civilisation, un monde que King taxe négativement. Le romancier montre dans une perspective naturaliste l'emprise d'une société de consommation uniforme conditionnée par une culture de masse, où prime le pouvoir, la violence et le matérialisme, pour nous donner le sentiment d'un cauchemar partagé.

Et si le destin sombre de Cujo se donne à lire dans le rapport de l'animal à la civilisation, il est aussi déterminé par le passage d'un milieu à un autre que représente la lisière de la forêt. Le roman de Stephen King joue ainsi sur la tension d'un espace liminal, afin de montrer la porosité entre le civilisationnel et le sauvage, le rationnel et le chaotique. De fait, c'est le franchissement d'un seuil qui instaure la convergence du naturalisme et du gothique rural en fonction de données environnementales pour transformer un décor pastoral en enfer. Dans la forêt, comme le souligne William Cronon, "the boundaries between human and non human, between natural and supernatural, have always seemed less certain than elsewhere" (CRONON apud MURPHY, 2013, p. 2). Joe Camber qui possède une ferme isolée au bout de la Route municipale répare des autos dans sa grange transformëe en garage. Lorsqu'il a acheté cette ferme, personne ne lui a fait connaître l'existence d'un trou, une sorte de cavité naturelle non loin de la maison, que même son fils n'a pas remarqué lors de ses promenades dans la nature. Cujo a moins de chance que Brett car, désireux de chasser un lapin, il voit son destin se sceller dans ce trou invisible peuplé de "petites bêtes brunes insectivores" (KING, 1981, p. 34) dont il ne connaît pas l'espèce et qui sont porteuses d'un puissant virus. Trop gros de taille, le saint-bernard ne peut s'introduire qu'au tiers dans ce trou 
débouchant sur une grotte, et il se trouve dans un entre-deux qui rend sa position intenable. Il fait figure d'assaillant aux yeux d'une horde de chauve-souris qu'il piège dans leur environnement naturel. Il s'opère alors pour Cujo une première plongée dans l'horreur se traduisant par la noirceur, la confusion et la perte de points de repère. L'animal se voit piégé à son tour, ce qui nous semble faire référence au sous-genre du film d'éco horreur comme le montre cette citation:

Ses aboiements réveillèrent les chauves-souris. Elles étaient affolées. On avait envahi leur antre. Elles se précipitèrent toutes ensemble, en piaillant, vers la sortie. (...) Il aboya plus fort encore et tenta de mordre les formes qui piaillaient en voletant au-dessus de sa tête. Ses crocs se refermèrent sur une aile brun-noir (KING, 1981, p. 35).

Naturalisme et gothique rural convergent donc dans cet environnement biologiquement contaminé dont est victime un chien d'autant plus vulnérable, qu'il n'a jamais été vacciné de la rage par un vétérinaire.

Le gothique rural vient également servir en termes de données spatiales déterministes, toute la trajectoire qui précède l'affrontement final de Donna et de sa Pinto avec cette machine à tuer qu'est devenu Cujo. Le déterminisme du milieu s'imprègne en effet d'une fatalité horrifique que connote une nature de plus en plus oppressante, au fur et à mesure que la jeune femme et son fils se rapprochent de la grange de Joe Camber où ils souhaitent faire réparer la voiture. La connotation négative du monde rural est renforcée par la dégénérescence que la jeune femme y perçoit et que vient symboliser un enfant comme abandonné à lui-même : "Le petit jardin était envahi par des mauvaises herbes (...) Un gamin d'à peu près deux ans se tenait, le ventre gonflé dans l'allée; ses couches trempées avaient glissé et laissaient apparaître son pénis minuscule. (...) Donna sentit la chair de poule la parcourir en détaillant l'enfant" (KING, 1981, p. 216-217). On décèle dans cette description une anticipation de la dégradation physique du jeune Tad qui mourra de déshydratation après trois jours de supplice dans la voiture.

La lutte de Donna contre Cujo n'est pas seulement celle d'une femme contre un animal qui a la rage et qui veut la dévorer, comme s'il souhaitait se venger d'avoir été mis au monde pour souffrir. C'est aussi le combat de deux forces prédatrices, celles de la nature qu'incarne Cujo, et celle de la civilisation que symbolise la Pinto où la jeune femme est prisonnière. Entre l'animal et l'automobile, il y a l'écran de la vitre qui sert à projeter le spectacle de la violence comme dans un film: "Un instant plus tard, la tête déformée et couverte d'écume surgit de l'autre côté de la vitre évoquant un monstre de cinéma qui aurait décidé de donner au public un dernier frisson en sautant de l'écran dans la salle" (KING, 1981, p. 224). Cette mise 
en scène du regard apparaît des plus significatives car l'animal, en exerçant sa violence aveugle sur la vitre de la Pinto afin de la briser, projette paradoxalement l'image de son corps en souffrance. La dégradation naturaliste emprunte ici un motif horrifique qui est le retournement de la violence contre soi, parce que le besoin de mutilation en est aussi un d'automutilation. L'hybridation de l'animal et de l'automobile se traduit par l'incorporation de la tôle dans les chairs saignées à vif. Mutilé et dépouillè de son enveloppe corporelle, la bête devient cette matière sanglante et informe qui consacre visuellement son statut de monstre. Contaminée par la morsure de Cujo qui lui a massacré la jambe et ouvert le ventre, Donna se mue à son tour en un être hybride, l'archétype de la bête humaine, mais encore l'allégorie de la vengeance et la figure mythique de la Furie. Dans cette lutte à finir avec le monstre, elle représente aussi le trope de la "Final Girl" que l'on retouve dans les films d'horreur, en particulier le slasher film, où la dernière femme en vie est confrontée au tueur pour l'éliminer et préserver la mémoire de l'histoire. ${ }^{3}$ La vision naturaliste apparaît donc ici comme l'affirmation d'un monde de désolation dénué de toute âme où l'homme, dans la continuité de la thèse darwinienne, doit dévorer l'Autre afin de rester en vie et de préserver son espèce. La question que pose le roman est alors la suivante: Comment survivre et affirmer un libre choix moral dans un monde où sévit le Mal et la menace de destruction? Peut-être, comme le fait Donna, en cherchant à s'affirmer par la rage du désespoir contre la prééminence déterministe, quitte à s'autodétruire.

Tandis que l'hybridation des genres chez King participe à la construction soigneusement élaborée d'un récit horrifique ayant le déterminisme naturaliste pour fondement, chez Mirbeau elle sert au contraire à déconstruire le texte et à en nier toute finalité déterministe. King recourt au naturalisme pour cautionner son histoire d'horreur et lui conférer un caractère rationnel, alors que Mirbeau emprunte à l'horrifique pour conduire sa narration vers l'irrationnel et la détourner de toute forme d'explication logique. Comme on a pu le constater, la représentation de Cujo en est une soigneusement composée à l'aide d'un métissage des genres, afin de justifier la métamorphose de l'animal et son devenir monstre. La représentation de Dingo, par contre, en est une composite et son caractère polymorphe vise à l'effacement. Cujo et Dingo signifient tous deux l'altérité mais de manière différente: pour le premier, celle-ci est associée à l'affirmation grandissante de la monstruosité et, pour le

\footnotetext{
${ }^{3}$ Figure essentielle du slasher film la "Final Girl" suit un parcours qui la fait passer de victime à bourreau, dû au fait qu'elle réussit à supprimer le tueur qui la poursuit. Pour une explication plus élaborée du concept de "Final Girl" dans le slasher film, voir CLOVER, 1992, p. 216-220.
} 
second, à une impossible définition de soi puisque Dingo est appréhendé par la négative, n'étant ni "chien, ni loup"4 et ni "un chien ni un homme" (MIRBEAU, 2001, p. 814).

L'animal apparaît dès le début du roman comme une forme monstrueuse et pétrissable, "sorte de boule fauve et molle" (MIRBEAU, 2001, p. 637) dont l'apparition ressortit à l'horrifique, car il est suggèré que "cet être larvaire (...), encore noyé dans les limbes" (MIRBEAU, 2001, p. 638), pourrait venir de l'au-delà, des marges de l'enfer. Par ailleurs, toute une mise en scène propre au cinéma d'horreur et au genre gothique contribue à créer un climat d'angoisse autour de l'étrange boîte qui contient le présent envoyé au narrateur par un mystérieux inconnu. La description même de la boîte participe de cette atmosphère morbide par son "aspect funèbre" (MIRBEAU, 2001, p. 636). Non seulement ce début anticipe une fin lugubre, mais encore la feuille d'envoi qui accompagne le paquet et sur laquelle il est écrit "Chien vivant" (MIRBEAU, 2001, p. 636) augmente la stupeur du destinataire et crée un effet d'attente propre à l'horrifique. Cela donne lieu de la part du narrateur à des spéculations sinistres formulées sur le ton de l'humour macabre: “- Il n’eût plus manqué, parbleu, que ce chien fût un chien crevé... (...) De sombres histoires de résurrectionnistes me revinrent à l'esprit... Je pensai: - Si j'allais trouver dans cette boîte, au lieu d'un chien vivant, des tronçons de corps humain!” (MIRBEAU, 2001, p. 636). Alors que chez King, l'horreur se donne à lire au premier degré, elle engendre chez Mirbeau, comme en atteste l'ouverture de son récit, une lecture au second degré où le ludisme de la parole sert à désamorcer la terreur en la parodiant: "Je frissonnai pour la forme et aussi parce qu'il m'est agréable de frissonner" (MIRBEAU, 2001, p. 636). L'emprunt au genre horrifique chez Mirbeau est donc de l'ordre du recyclage et de la parodie.

La description de Dingo sur laquelle s'ouvre la narration fait écho à celle de Cujo à la fin du roman. Tous deux ont en effet en commun d'être affamés et abandonnés à eux-mêmes, entre la vie et la mort. Mais alors que l'un est un nouveau-né et que l'autre est devenu une vieille bête ratatinée, avec "un regard de vieillard atteint de la cataracte" (MIRBEAU, 2001, p. 417), ils se ressemblent par leur apparence terrifiante et informe qui atteste d'une conception cyclique de l'existence. Ainsi Mirbeau nous offre-t-il un portrait du jeune chiot Dingo similaire à celui d'un homme âgé:

Figurez-vous un museau de vieux petit fonctionnaire (...) tout plissé de mauvaise humeur; une tête beaucoup trop grosse, beaucoup trop lourde pour le corps; un corps vaguement ébauché; des yeux à peine ouverts, à peine

\footnotetext{
${ }^{4}$ Sir Edward Herpett dit à ce sujet: "J'ai appris à Melbourne, d'un Hollandais professeur de langue malaise, que Dingo est un vocable nègre qui, précisément, signifie: ni chien, ni loup” (MIRBEAU, 2001, p. 641).
} 
visibles dans la fente des paupières boursouflées. Sur le ventre rose, plein, glabre, tacheté de roux, un reste séché de cordon ombilical se tortillait comme un ver... (MIRBEAU, 2001, p. 637).

Dingo et Cujo ont aussi en commun d'avoir un corps souillé de déjections inspirant la répulsion. Il renvoie à cette forme d'évidement qui caractérise le corps horrifique et fait se rencontrer le dedans et le dehors. Ainsi la nature anormale ou répulsive du corps appelle une vision basée sur l'excès, qui fait basculer nos repères et nous plonge dans l'indétermination. Mirbeau conçoit cette indistinction en termes artistiques puisqu'il rattache le corps en mutation à une esquisse vouée à l'effacement. ${ }^{5}$ Que ce soit par le biais d'une référence directe à la peinture ou au cinéma, Dingo et Cujo sont tous deux représentés en fonction d'une rhétorique où le visuel spectaculaire vise à la fois à choquer et émouvoir. Mais tandis que la représentation de Cujo est enracinée dans un cadre réaliste, celle de Dingo s'en éloigne au contraire, pour en faire dès le début du roman un être d'exception, à caractère surnaturel. Il survit en effet mystérieusement à un voyage qui aurait dû le conduire à la mort.

À cet égard le tableau qui est fait de Dingo apparaît davantage comme une construction imaginaire, même s’il est imprégné de lectures scientifiques. Le personnage du savant excentrique que représente Sir Edward Herpett, nous livre non sans humour, dans la lettre qu'il écrit au narrateur, une tentative de classification naturaliste du dingo en fonction des notions d'espèce et de milieu. Il emprunte essentiellement aux théories évolutionnistes en vogue à l'époque, faisant valoir une mutation biologique du monde vivant et une adaptation des espèces aux milieux qui prend la forme d'une lutte pour la survie. C'est ce que représente sur le mode fantaisiste, voire fantastique, l'adaptabilité du chiot Dingo qui, éloigné des espaces sauvages infinis de l'Australie, réussit à survivre dans une boîte scellée. Le registre naturaliste visant à enfermer l'animal dans une définition classificatoire apparaît d'autant plus forcé, que le dingo résulte d'un métissage et que l'espèce à laquelle il appartient est vouée à la mouvance et à la disparition. Cette extinction probable opère un glissement du discours scientifique vers un ton lyrique qui mine l'idée de progrès et de civilisation, pour laisser poindre une nostalgie de l'état sauvage. La description composite du chien contribuant à en faire un être inclassable, le réduit au bout du compte à une force vitale primitive ne pouvant s'expliquer, tant elle est le signe manifeste d'une incohérence. De plus, les divers registres et modes selon lesquels s'opère une tentative de description du dingo, sont en dernier lieu

\footnotetext{
${ }^{5}$ Comme le souligne Enda Mc Caffrey, "Dingo, en tant que chien exotique, personnifie la variété de la nature, l'équivalent de l'hybridité dans la représentation esthétique. (...) Dingo, dans sa représentation corporelle, incarne une forme de mouvement qui va contre les demandes symétriques et conventionnelles de l'École des Beaux-Arts, et participe d'une conception de l'art qui est moderne, différente et progressiste" (Cahiers Octave
} Mirbeau, n. 7, 2000, p. 72). 
désamorcés par la prééminence de l'horrifique qui enfreint tout discours pour faire de l'animal une présence silencieuse et invisible. Cette présence fait d'autant plus peur que le dingo ne laisse aucune trace de son passage et qu'il n'aboie jamais. Les hurlements qu'il émet rarement font taire la parole pour laisser place à la terreur:

Je vous assure, cher ami, que ce hurlement qu'il m'a été donné deux fois d'entendre à la nuit tombante, au bord du désert rouge, alors que le vent faisait siffler comme des locomotives l'écorce arrachèe des eucalyptus et que dans les arbres le lampfing jacass riait de son rire démoniaque, est mille fois plus sinistre que le hurlement des loups. J'ai connu là des minutes d'effroi que plus jamais je ne pourrai oublier (MIRBEAU, 2001, p. 642).

Ainsi Dingo se distingue de Cujo qui, bien qu'habité par une altérité horrifique dans sa représentation visuelle, peut au départ être clairement défini en termes réalistes, le saintbernard étant un chien de race facilement reconnaissable, un animal familier dont le comportement est voué au sacrifice. Et c'est paradoxalement cette dimension sacrificielle qui consacrera l'inéluctable horreur du destin de l'animal. Mirbeau, au contraire, recourt à une non-définition du dingo comme un chien qui n'en est pas un, il le complexifie, le défamiliarise pour mettre en avant une liberté de comportement pouvant transgresser les interdits et atteindre le comble de l'horreur. Et lorsque, chez King, le doux regard du chien saint-bernard devient vide par la suite, c'est pour nous permettre de lire de façon tangible, dans les yeux de l'animal, l'étrangeté qui le hante comme un mal. Par contre quand le narrateur de Mirbeau plonge dans les ténèbres des yeux de Dingo, il réagit à leur "inexpression hallucinante" (MIRBEAU, 2001, p. 664) par la prise de conscience de son impuissance à l'interpréter. Cette impossibilité d'interprétation apparaît aussi comme une façon de contrecarrer toute forme de dèterminisme naturaliste, Mirbeau étant de ceux qui pensent que l'hérédité et le milieu sont des principes auxquels on peut échapper.

Pour en faire la démonstration, il fait utiliser à son narrateur des méthodes d'enseignement qui correspondent à ses idées et visent à civiliser Dingo, à lui inculquer des habitudes de vie humaine. C'est toutefois une vision contre-nature qui nous est proposée de l'animal puisque le voilà artificiellement devenu un chien de maison bourgeoise, bien que son maître le considère comme une bête sauvage n'appartenant à aucune civilisation et exempte de toute corruption. Transplanté dans un milieu raffiné et voluptueux qui est l'inverse de son environnement naturel, Dingo plonge avec délice dans ce nouveau décor, dans cette débauche de matières précieuses, non pas pour exprimer sa docilité comme le suggère les apparences, mais plutôt pour affirmer son goût de la provocation et ses appétits nouveaux. Ne développet-il pas une forme de jouissance singulière dans la comestibilité du beau et de l'ersatz? Cette 
esthétisation décadente du dingo est d'ailleurs corroborée par la description que le savant Herpett fait de l'animal: "Ces animaux, continuait-il sont extraordinaires et magnifiques. Vêtus d'or et de feu, avec des dessous de bistre clair, (...) la queue touffue, traînant à terre majestueusement, comme un gros boa de zibeline (...)" (MIRBEAU, 2001, p. 641). Les préceptes naturalistes et décadents sont donc mis dos à dos pour dèsamorcer en un ludisme textuel toute vision essentialiste du chien. L'animal s'affirme au contraire dans sa mouvance, dans la fluctuation de ses comportements, qui lui permettent d'affiner sa personnalité contre toute forme d'atavisme ou de discours pédagogique. Le maître lui-même le confirme en disant: "Heureusement, Dingo, étant plus intelligent que moi, résistait" (MIRBEAU, 2001, p. 657). Les rôles de professeur et d'élève finissent par s'inverser au fur et à mesure de la progression du roman, au point que le narrateur s'efface peu à peu pour laisser la place au chien.

Ce dernier décide d'entreprendre à son tour l'éducation éthologique de la chatte, Miche, pour la rendre sauvage, la transformer en fauve. Le passage d'une espèce à l'autre, qui est signe d'évolution et relève de la nature, est donc ici caricaturé par une inversion contrenature visant non pas le progrès mais une régression à caractère décadent. La leçon consiste à faire franchir à la chatte un seuil, celui de la lisière du bois qui l'amène dans un monde inconnu de sauvagerie chargé d'une dimension horrifique:

Miche miaulait toujours et, secouée de frissons, les prunelles tout ouvertes, elle regardait à sa droite, à sa gauche, devant, derrière elle, et sans doute elle pensait qu'elle était bien petite, bien trop petite pour ce grand espace dont Dingo lui ouvrait les perspectives infinies, inconnues, effrayantes (MIRBEAU, 2001, p. 792).

Cette escapade de Miche en compagnie de Dingo renvoie de prime abord à celle de Cujo dans la campagne, car elle fait figure d'un divertissement pouvant basculer dans le cauchemar. Mais Dingo contrairement à Cujo ne se laisse pas entraîner par le destin dans un gouffre qui se confond avec la nature. Il dirige sciemment Miche vers un endroit caché qu'il est seul à connaître, afin de lui apprendre à tuer. Non seulement cette leçon pratique a-t-elle pour but de parodier l'influence de l'hérédité et du milieu en rapport avec le concept d'expérimentation, mais encore permet-elle d'ètaler un goût pour le meurtre et le plaisir de faire "gicler le sang avec la vie" (MIRBEAU, 2001, p. 796), comme dans un film d'horreur. De telles constatations confirment que les détournements génériques ont non seulement pour but chez Mirbeau de saper la causalité naturaliste du texte, mais encore de mettre en avant sa part d'invention et de fantaisie par le biais du recyclage. 
Une autre façon de transcender le naturalisme est de pousser l'horreur à ses limites, de nous entraîner dans une spirale de violence qui s'amplifie et se propage afin que se prolonge le divertissement de la peur. Ainsi les échappées sauvages de plus en plus fréquentes de Dingo nous semblent-elles obéir à un scénario de film d'horreur où l'animal s'adonne avec joie à un jeu de massacre qui en fait une sorte de monstre tueur invisible qu'il faut traquer et abattre pour obtenir vengeance. ${ }^{6}$ Dingo revêt alors une dimension à la fois fantasmatique et fantastique puisqu'il est associé à un être mystérieux, une force inconnue, un fléau sur terre, une incarnation du diable qui terrorise tout le monde et provoque l'exaltation sanguinaire des paysans de Ponteilles-en-Barcis. Ce village où habite le narrateur et son chien, situé à proximité du bois renvoie tout comme Castle Rock dans Cujo, au sous-genre horrifique du gothique rural, ne serait-ce qu'en raison de la dégénérescence des ses habitants comparés par Mirbeau à des "peuplades primitives sauvages" (MIRBEAU, 2001, p. 666). Ponteilles-enBarcis est en effet peuplé de quelques bourgeois corrompus et d'un grand nombre de paysans que le narrateur du roman qualifie en ces termes:

(...) population inquiète, sournoise, hargneuse et blême, sur qui pèse, depuis d'immémoriales années, un sinistre héritage de dèchèance alcoolique et de tuberculose. Les visages creusés, où sur les pommettes fleurissent les fleurs pourprées, les fleurs rosâtres de la pourriture ou de la mort (MIRBEAU, 2001, p. 667).

Pour ces gens, Dingo apparaît comme un animal étrange, dont le comportement ne présage rien de bon. Mais l'intérêt de cet animal vient justement de sa différence, de ces mélanges dissociés qui le constituent et sur lesquels on n'a aucune prise. La nature sauvage de Dingo, c'est aussi son caractère indomptable, sa violence insaisissable, ce par quoi il affirme son indépendance contre toute loi de la nature ou de la société. Pour Dingo façonner sa personnalité revient à franchir des zones d'ombre, à revendiquer une opacité qui échappe à l'autre et devient sa liberté. Et tandis que Cujo fuit la lumière afin de mieux cacher le mal qui l'accable, Dingo disparaît dans la forêt pour exulter dans une ivresse barbare qui n'appartient qu'à lui: "Mais Dingo se leva sans hâte, se dirigea vers le petit mur du fond, qui donnait sur le bois et, le franchissant d'un bond léger, il disparut sous les feuilles, dans le taillis" (MIRBEAU, 2001, p. 775). Le caractère elliptique de ses fuites et le fait qu'il est invisible et

\footnotetext{
${ }^{6}$ Comme le rappelle à juste raison Barry Langford, le film d'horreur implique une vengeance ritualisée contre l'intrus, le monstre prédateur, tandis que dans le film de science-fiction, la revanche est rationalisée: "Violence, to be sure, may play a ubiquitous role in defeating the intruder and restoring 'normality', but the violence of the SF film is far more likely to be ostensibly rational and considered, that of the horror film, ritualized and reactive (the pogrom-like revenge of the villagers with their flaming torches)" (LANGFORD, 2005, p. 164).
} 
silencieux, bien que laissant derrière lui une véritable boucherie, contribuent à conférer à l'animal tueur une dimension surnaturelle et effrayante.

L'acte de tuer est par ailleurs mis en spectacle sur un mode jubilatoire horrifique, qui consiste à décliner visuellement et à amplifier les diverses atrocitès commises par cette force invisible qui fait des ravages sur son passage:

Toutes les poules étranglées, éventrées, toutes les poules mortes (...), déjà raidies, étaient rangées, comme pour une exposition, côte à côte, méthodiquement (...) Il y en avait cent. Les unes semblaient intactes, sauf leurs plumes qui étaient farouchement rebroussées et mouillées de bave sanglante. Des autres aplaties, tordues, en bouillie, les entrailles sortaient et dévidaient sur le sable leurs anneaux ronds. (...) On eût dit qu'une grande force de destruction, un formidable cyclone, une invasion de bêtes forcenées avaient passé par là (MIRBEAU, 2001, p. 773-774).

Cette plongée dans l'horreur chez Mirbeau consiste donc en une récupération des instincts sauvages au profit du spectaculaire et de la création artistique. À cet égard, la lutte de l'animal contre toutes les embûches des escarpes dans ce "décor de théâtre" (MIRBEAU, 2001, p. 814) qu'est devenu Paris, se transforme en spectacle où la force combative de Dingo acteur fait la joie des spectateurs. Cette spectacularisation de la violence ressortit également à son caractère non sélectif qui anarchise les espèces et fait se confondre nature et artifice, comme on peut le constater avec la scène du massacre des manteaux de fourrure par Dingo: “Les fourrures sont des bêtes vivantes, elles ont gardé l'odeur de la bête vivante. Dingo les déchiquette, les balance et les traîne. Sans doute le sang va jaillir et aussi les viscères gluants et mous (...)" (MIRBEAU, 2001, p. 829). Et le narrateur d'ajouter à ce sujet: "Je ne ferai pas l'énumération de tous ses massacres. Ils furent nombreux et affreux, parfois d'un comique imprévu, d'une irrésistible fantaisie" (MIRBEAU, 2001, p. 831). Faut-il en conclure que le roman de Mirbeau convoque un scénario horrifique où la violence vouée à la répétition finit par se parodier elle-même? Ajoutons à cela que dans Dingo, à l'opposé de Cujo, il y a un refus d'expliquer le comportement effrayant du chien lorsqu'il plante ses crocs dans la gorge d'un humain, par une éventuelle maladie de la rage. Le vétérinaire nie l'existence de cette maladie et la réduit à une simulation avec des contractions convulsives, ce qui renvoie une fois de plus au récit horrifique: "Les chiens n'ont pas la rage... (...) Ils ont une affection épileptiforme qui la simule... (...) Ils ont tous les symptômes de l'épilepsie; ils bavent... ils se mordent la langue... ils ont des convulsions. Et c'est tout...” (MIRBEAU, 2001, p. 826).

Chez Mirbeau la manifestation du mal ne saurait donc être envisagée en termes d'atavisme car Dingo, dans l'entre-deux de son animalité et de son humanité, échappe à toute forme de rationalisme et demeure libre de ses actes. Une telle étude confirme que l'horrifique

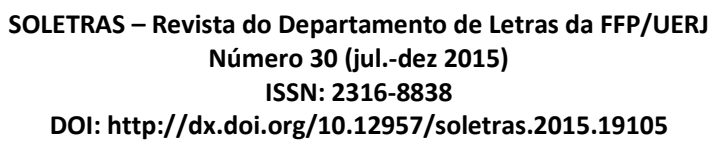


chez Mirbeau sert à déconstruire le naturalisme et à en faire vaciller les assises. Si Dingo succombe à sa race et à son milieu, c'est parce qu'il affirme sa volonté de les renier par une sensibilité qui n'appartient qu'à lui et qui est de l'ordre de l'irrationnel. Il revient non pas au narrateur mais à l'animal de faire le choix de sa propre fin - celle de sceller son attachement à sa maîtresse par la mort. Dingo impose ainsi son histoire contre une autre possible - un récit d'horreur où il serait torturé à mort pour cause de carnage. En fin de compte ces deux scénarios sont renvoyés dos à dos afin d'en souligner l'arbitraire et de laisser le texte ouvert. C'est aussi une liberté créatrice qui s'affirme à travers Dingo, tandis que Cujo, lui, n'a d'autre choix que d'être éliminé pour obéir aux lois du genre horrifique, d'autant plus contraignantes qu'elles sont assujetties à un naturalisme où priment les nécessités du réel.

\section{Références bibliographiques :}

ANCET, Pierre. Phénoménologie des corps monstrueux. Paris : PUF, 2006.

AUMONT, Jacques. Du Visage au cinema. Paris: Éditions de l’Étoile, 1992.

BADLEY, Linda. Writing Horror and the Body. The Fiction of Stephen King, Clive Barker, and Anne Rice. Westport: Greenwood Press, 1996.

CLOVER Carol. Men, Women and Chain Saws: Gender in the Modern Horror Film. Princeton: Princeton University Press, 1992.

GORMAN, James \& KENNEALY, Christine. "Australia's Changing View of the Dingo". New York Times, 06 March 2012.

KING, Stephen. Cujo. Trad. Natalie Zimmermann. Paris: Albin Michel, 1981.

LABOR, Earle. "Jack London's Mondo Cane: 'Bâtard,' The Call of the Wild, and White Fang”. In: TAVERNIER-COURBIN, Jacqueline (dir.) Critical Essays on Jack London. Boston: G.K. Hall, 1983.

LANGFORD, Barry. Film Genre: Hollywood and Beyond. Edinburgh: Edinburgh University Press, 2005.

MC CAFFREY, Enda. "Le Portrait d'un artiste en jeune chien : Incarnation et mouvement dans Dingo". Cahiers Octave Mirbeau. Angers : Société Octave Mirbeau, n. 7, 2000.

MIRBEAU, Octave. Euvre romanesque, vol. III. Paris: Buchet/Chastel/Société Octave Mirbeau, 2001.

MURPHY, Bernice M. The Rural Gothic in American Popular Culture. Backwoods Horror and Terror in the Wilderness. London: Palgrave Macmillan, 2013. 
STRENGELL, Heidi. Dissecting Stephen King: From the Gothic to Literary Naturalism. Madison: The University of Wisconsin Press, 2005.

WINTER, Douglas. Stephen King: The Art of Darkness. New York: New American Library, 1986.

\section{Desvios naturalistas e horríficos em Dingo de Octave Mirbeau e Cujo de Stephen King}

Resumo: Dingo (1913) de Octave Mirbeau e Cujo (1981) de Stephen King têm em comum o fato de apresentarem como heróis um cão cuja transmutação subversiva se efetua em função de dados naturalistas e horríficos. Embora ambos sejam cães matadores, sua violência assassina se baseia no regime do livre-arbítrio para um e no determinismo para o outro. $\mathrm{O}$ objetivo desse estudo é mostrar, pelo viés da representação desses dois cães, que o horrífico em Mirbeau serve para desconstruir o naturalismo e abalar seus fundamentos. Se Dingo sucumbe à sua raça e a seu meio, é porque ele afirma sua vontade de renegá-los. Logo, é ele, mais do que o narrador, que faz a escolha de seu próprio fim e impõe sua história contra uma outra possível, uma narrativa de horror na qual ele seria torturado por carnificina. Desse modo, é também uma liberdade criativa que se afirma através de Dingo, enquanto Cujo não tem outra a escolha a não ser aquela de ser eliminado para obedecer as leis do gênero horrífico, ainda mais severas em Stephen King por serem sujeitas a um naturalismo no qual prevalecem as necessidades do real.

Palavras-chave: Determinismo. Naturalismo. Paródia. Octave Mirbeau. Stephen King.

Recebido em: 5 de julho de 2015.

Aprovado em: 15 de novembro de 2015 . 